\title{
VERITAS (Venus Emissivity, Radio Science, InSAR, Topography And Spectroscopy): A Proposed Discovery Mission
}

Suzanne Smrekar1, Scott Hensley1, Darby Dyar2, Jörn Helbert3, and the VERITAS Science Team, (1) Jet Propulsion Laboratory, Caltech, 4800 Oak Grove Dr., Pasadena, CA USA (ssmrekar@jpl.nasa.gov, shensley@jpl.nasa.gov), (2) Dept. of Astronomy, Mount Holyoke College, South Hadley, MA USA (mdyar@mtholyoke.edu), (3) Inst for Planetary Research, DLR, Rutherfordstrasse 2, 12489 Berlin, Germany (Joern.Helbert@dlr.de)

\begin{abstract}
VERITAS addresses one of the most fundamental questions in rocky planetary evolution: why did these twin planets diverged down different evolutionary paths? Venus may hold lessons for past and future Earth. Venus' hot lithosphere may be a good analog for early Earth, and could be responsible for the apparent lack of plate tectonic [1]. Determining the factors that lead to the initiation of plate tectonics would inform our predictions for rocky Earth-sized exoplanets. VERITAS answers key questions about Venus' geologic evolution, determine what processes are currently active, and search for evidence for past or present water. VERITAS would launch in 2026.
\end{abstract}

\section{Introduction}

VERITAS addresses one of the most fundamental questions in rocky planetary evolution: why did these twin planets diverged down different evolutionary paths? Venus may hold lessons for past and future Earth. Venus' hot lithosphere may be a good analog for early Earth, and could be responsible for the apparent lack of plate tectonic [1]. Determining the factors that lead to the initiation of plate tectonics would inform our predictions for rocky Earth-sized exoplanets. VERITAS answers key questions about Venus' geologic evolution, determine what processes are currently active, and search for evidence for past or present.

\section{Payload}

The VISAR X-band [2] measurements include: 1) a global digital elevation model (DEM) with $250 \mathrm{~m}$ postings, $5 \mathrm{~m}$ height accuracy, 2) Synthetic aperture radar (SAR) imaging at $30 \mathrm{~m}$ horizontal resolution globally, 3) SAR imaging at $15 \mathrm{~m}$ resolution for targeted areas, and 4) surface deformation from RPI at $2 \mathrm{~mm}$ precision for targeted, potentially active areas.

VEM [3,4] will produce surface coverage of most of the surface in 6 NIR bands located within 5 atmospheric windows and of 8 atmospheric bands for calibration and water vapor measurements.

VERITAS will use Ka-band uplink and downlink to create a global gravity field with 3 mgal accuracy / $145 \mathrm{~km}$ resolution (130 spherical harmonic degree and order or $\mathrm{d} \& \mathrm{o}$ ) and providing a significantly higher resolution field with much more uniform resolution than that available from Magellan.

\section{Science}

\subsection{Geologic Evolution}

VERITAS answers key science questions via: 1) examining the origin of tesserae plateaus -possible continent-like features, 2) assessing the history of volcanism and how it has shaped Venus' young surface, 3) looking for evidence of prior features buried by volcanism, and 4) determining the links between interior convection and surface geology. In particular, VERITAS will examine the stratigraphy and nature of tesserae deformation features, determine the processes modifying impact craters, search for evidence of pre-existing features such as buried impact basins, and determine the origin of tectonic features such as huge arcuate troughs that have been compared to Earth's subduction zones. 
Magellan spherical harmonic gravity field has an average resolution of only $550 \mathrm{~km}$ [5], which is too low to determine elastic thickness [6]. VERITAS data, with an average resolution of 165 $\mathrm{km}$, will enable estimation of elastic thickness (a proxy for thermal gradient) and resolution of specific geologic processes [7].

\subsection{Water and Igneous Rock Type}

VERITAS looks for the chemical fingerprint of past water in the form of low Fe, high Si rock in the tessera plateaus [8] and larger tesserae inliers, and for present day volcanic outgassing of volatiles in the form of near surface water variability associated with recent or active volcanism.

\subsection{Current Activity}

Several studies have found evidence of current or recent volcanism on Venus. [e.g. 9]. VERITAS uses a variety of approaches to search for present day activity, including 1) tectonic and $\mathrm{cm}$-scale volcanic surface deformation, 2) chemical weathering, 3) thermal emission from recent or active volcanism, 4) topographic or surface roughness changes, and 5) comparisons to past mission data sets.

\section{Summary and Conclusions}

VERITAS will create a rich data set of high resolution topography, imaging, spectroscopy, and gravity. These co-registered data will be on par with those acquired for Mercury, Mars and the Moon that have revolutionized our understanding of these bodies. In addition to answering fundamental science questions, VERITAS' data would motivate further Venus missions. Global topography is needed to enable both clutter removal for subsurface sounding, and as part of the data reduction for VEM at tessera. Active surface deformation would promote a seismic mission. Accurate topography plus surface rock type would optimize targeting of surface or areal missions.

\section{Acknowledgements}

A portion of this research was conducted at the Jet Propulsion Laboratory, California Institute of Technology, under contract with NASA.

\section{References}

[1] D. Bercovici and Y. Ricard, "Plate tectonics, damage and inheritance," Nature, vol. 508, p. 513, 2014.

[2] S. Hensley, J. Martin, S. Oveisgsharan, X. Duan, and B. A. Campbell, "Radar Performance Modeling for Venus," VEXAG Meeting, Applied Physics Laboratory, 2018.

[3] J. Helbert, D. Dyar, I. Walter, D. Wendler, T. Widemann, E. Marcq, et al., "The Venus Emissivity Mapper (VEM): obtaining global mineralogy of Venus from orbit," in infrared Remote Sensing and Instrumentation XXVI 2018, p. 99730R.

[4] Helbert, J. et al., Spectroscopy of the surface of Venus - in the laboratory and from orbit, abstract, this meeting, 2019.

[5] Konopliv A., Banerdt, W.B., and W.L. Sjogren, Venus Gravity: 180th Degree and Order Model, Icarus 139, 3-18, 1999.

[6] M. A. Wieczorek, Gravity and Topography of the Terrestrial Planets, Treatise on Geophysics, vol. 10, pp. 165-206, 2007.

[7] Andrews-Hanna J. C. Smrekar S. E. Mazarico, Venus Gravity Gradiometry: Plateaus, Chasmata, Coronae, and the Need for a Better Global Dataset LPSCXLVII Abst. \#2907, 2016.

[8] Dyar, M.D. et al., Leveraging Rock Type, Mineralogy, and Oxidation State from Six-Window Emissivity Spectra at $440 \mathrm{C}$, abstract, this meeting, 2019

[9] S. E. Smrekar, E. R. Stofan, N. Mueller, A. Treiman, L. Elkins-Tanton, J. Helbert, et al., "Recent Hot-Spot Volcanism on Venus from VIRTIS Emissivity Data," Science, 2010.Smrekar et al. (2010) Science 